\title{
Papers
}

\section{Lessons for the laboratory from a general practitioner survey}

\author{
A M Boyde, R Earl, S Fardell, N Yeo, J M Burrin, C P Price
}

\begin{abstract}
Aims-To assess the current performance of the clinical biochemistry service provided to general practitioners, with particular attention to result turnround times, and to identify any improvements required.

Methods-Postal questionnaire survey of general practitioners in the London Borough of Tower Hamlets who used the clinical biochemistry laboratory of the Royal London Hospital. A flow analysis study of turnround times for general practitioner samples was also performed.
\end{abstract}

Results-Responses to the questionnaire showed that although $82 \%$ of general practitioners thought the current quality of service provided was better than fair, the actual turnround times achieved were longer than the acceptable times required. There was also a strong demand $(>66 \%$ of responders) for additional informationsuch as highlighting of abnormal results-to be provided with results. There was wide variability between practitioners in their use of the laboratory (from none to $>800$ requests per year), with no apparent correlation to practice size. Of the repertoire of tests requested, a surprisingly high percentage $(14.3 \%)$ were for thyroid function. Flow analysis of turnround times for thyroid function tests showed that problems lay not with the time taken for analysis (only $7.8 \%$ of the total turnround time) but with the preand postanalytical phases, that is, the sample collection and results delivery service.

Conclusions-Increasing the proportion of health care delivered in the primary care sector will inevitably increase the requirement for pathology services. Improvements in the specimen collection and results delivery service to general practitioners are needed to meet their expectations. It remains to be determined whether increased investment in these aspects of laboratory service would result in improved patient care in the primary sector.

(F Clin Pathol 1997;50:283-287)
Keywords: general practitioners; pathology services; performance survey

The health service reforms, which sought to increase the proportion of health care delivered in the primary care sector and provided the opportunity for general practitioners to be budget holders for certain areas of work, have led to increasing interest in the requirement for pathology services in the primary care sector. ${ }^{1}$ The two major issues that have evolved for pathology from this development are the style and repertoire of services required and the possibility that some of these services might be provided directly within the primary care environment. $^{2}$

Historically, the needs of hospital consultants have taken priority over those of general practitioners so far as many pathology services are concerned. Little attention has been paid to ensuring that communications between the requester and the provider really met the needs of the general practitioners, and poor specimen collection services and reporting arrangements are commonplace in the primary care sector..$^{3-6}$ On the other hand, it might be claimed that the general approach to organisation of primary care practice did not demand a rapid response from the laboratory, although the telephoning of abnormal results to the surgery is in line with common laboratory practices, thus ensuring an early warning to all clinicians when rapid action is needed.

New developments in laboratory technology have improved turnround times, particularly for endocrine analyses such as thyroid function tests. These tests are increasingly being requested by general practitioners as care of patients is transferred back to the primary sector, and it has been recognised that the proportion of endocrine investigations required by general practitioners is higher than for hospital doctors. ${ }^{4}$

The additional new dimension to diagnostic testing in the primary care sector has been the development of small and simple, often disposable, devices that allow testing near to the patient. Thus in certain instances general practitioners can now also decide to undertake tests in their surgeries. ${ }^{2-10}$ 
Table 1 Data from questions related to overall quality of service, and problems with collection of samples or return of reports (rated on a score 1-5 from excellent-poor, always-never, or agree-disagree as appropriate)

\begin{tabular}{llrrrrr}
\hline & \multicolumn{3}{l}{ Number of respondents } & & \\
\cline { 2 - 7 } Issue & \multicolumn{1}{l}{ N } & 2 & 3 & 4 & 5 & No answer \\
\hline Quality & 6 & 35 & 15 & 6 & 0 & 6 \\
Inappropriate collection time & 8 & 15 & 9 & 21 & 11 & 4 \\
Collection too infrequent & 3 & 11 & 11 & 19 & 19 & 5 \\
No specific collection time & 2 & 4 & 11 & 13 & 31 & 1 \\
Results return time acceptable & 3 & 23 & 25 & 10 & 3 & 4 \\
\hline
\end{tabular}

These developments pose some very important questions for the laboratory director with regard to the repertoire and response times required. In order to gather opinions and data to answer some of these questions, two surveys were undertaken, one assessing the use of a clinical biochemistry service offered to a group of general practitioners and the other a study of factors influencing turnround times of endocrine results to general practitioners following the introduction of an automated immunoassay analyser in the laboratory.

\section{Methods}

To assess usage of the clinical biochemistry service, a questionnaire was sent to 106 general practitioners in the London Borough of Tower Hamlets after piloting with three practitioners and incorporating modifications based on their response. The questions dealt with (1) general satisfaction with the service, (2) frequency of collection problems, (3) detail of report response times, and (4) opinion on additional information provided with reports. In addition, data were collected from the laboratory on the use of the clinical biochemistry service by the doctors who responded, and for the group overall.

The second study involved an audit of the rate of progress of blood samples for endocrine analyses from the time the general practitioner requested the test to the arrival of the results back at the practitioner's office. The endocrine analyses studied were those which were available on the Immulite (EURO DPC Ltd, United Kingdom) automated immunoassay analyser. This study also involved a questionnaire sent to 17 general practitioners in the London borough of Tower Hamlets assessing desirable turnround times for endocrine analyses. The practitioners questioned were those who were regular users of the endocrine service provided by the clinical biochemistry laboratory of the Royal London Hospital.

\section{Results}

The service provided at the time of this questionnaire involved the use of a request form to be sent with the sample, and a single collection of samples during the day using a van that visited health centres consecutively over a period of five hours. All samples were delivered simultaneously to the laboratory at the end of the run, and reports were returned on a daily basis using the same transport arrangement. Grossly abnormal results would be telephoned to the relevant general practitioner or health centre immediately after validation in the laboratory.

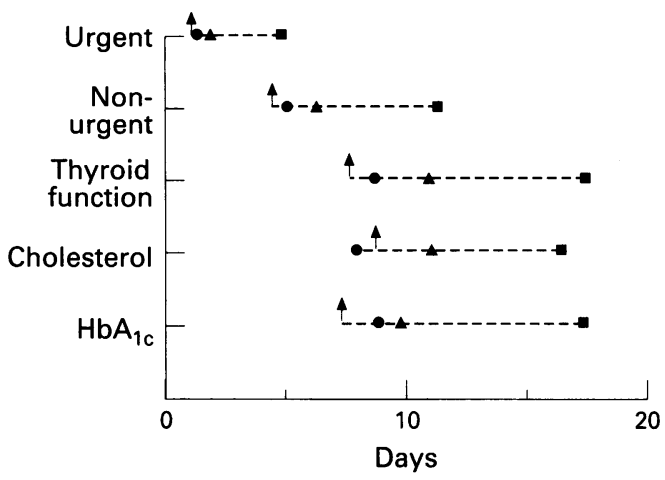

Figure 1 Data on the best ( $\bullet$ ), average ( $\mathbf{\Lambda}$ ), and worst (i) turnround times for a range of biochemical tests from dispatch of sample to receipt of report, together with the maximum acceptable turnround time (1).

USER SURVEY

There were 68 respondents in the survey (64\%). Respondents were asked to express their degree of satisfaction in the service by scoring between 1 (positive) and 5 (negative); the data are shown in table 1. Respondents were asked to indicate their current views on the overall quality of service including results return time and to comment specifically on problems occurring with specimen collection. The data indicate a high degree of satisfaction with the quality of the results produced and furthermore show that the results were generally returned within an acceptable time frame. However, the data show clear dissatisfaction with the aspect of specimen collection addressed by the survey, namely the frequency and actual timing of specimen collection.

Opinions were sought on expectation of, and experience with, turnround times from dispatch of sample to receipt of result for a range of investigations: (a) urgent, (b) non-urgent, (c) thyroid function test, (d) cholesterol, and (e) $\mathrm{HbA}_{\mathrm{ic}}$ against four criteria: (i) usual experience, (ii) best, (iii) worst, and (iv) maximum acceptable. Despite the apparent satisfaction with the service (as shown in table 1), the data shown in figure 1 indicate that the actual turnround times achieved were longer than the acceptable times in most cases.

Opinions were sought on the provision of additional information with a report. The data are shown in table 2 . The responses indicated a clear requirement for additional information, particularly the provision of reference ranges and the highlighting of abnormal results.

The total workload for the year 1993-94 requested by the 106 general practitioners surveyed is shown in table 3 . The number of requests plotted against the general practitioner's list size are shown in figure 2; the list size for each practitioner was determined by divid- 
Table 2 Data on requirement for additional information on report forms scored 1-5 from very important to unimportant. Values are numbers of responses in each score category

\begin{tabular}{|c|c|c|c|c|c|c|}
\hline \multirow[b]{2}{*}{ Service } & \multicolumn{5}{|c|}{ Score } & \multirow[b]{2}{*}{ No answer } \\
\hline & 1 & 2 & 3 & 4 & 5 & \\
\hline $\begin{array}{l}\text { Normal ranges } \\
\text { Abnormal result highlighted }\end{array}$ & $\begin{array}{l}45 \\
46\end{array}$ & $\begin{array}{r}11 \\
4\end{array}$ & $\begin{array}{l}3 \\
6\end{array}$ & $\begin{array}{l}4 \\
4\end{array}$ & $\begin{array}{l}4 \\
2\end{array}$ & $\begin{array}{l}1 \\
2\end{array}$ \\
\hline
\end{tabular}

Table 3 Test requesting pattern for general practitioners using Royal London Clinical Biochemistry Laboratory (April 1993 to March 1994)

\begin{tabular}{lcc}
\hline Test & $\begin{array}{l}\text { Number of } \\
\text { requests }\end{array}$ & \% Total GP workload \\
\hline Urgent & 42 & 0.1 \\
U\&E and LFT & 11581 & 31.4 \\
$\begin{array}{l}\text { Thyroid function } \\
\text { test }\end{array}$ & 5258 & 14.3 \\
Glucose (inc & & \\
$\quad$ OGTT) & 6486 & 17.6 \\
Cholesterol & 1874 & 5.1 \\
HbA & 1702 & 4.6 \\
Other non-urgent & 4973 & 13.5 \\
Total & 36889 &
\end{tabular}

$\mathrm{U} \& \mathrm{E}=$ urea and electrolytes; LFT=liver function tests; OGTT=oral glucose tolerance test.

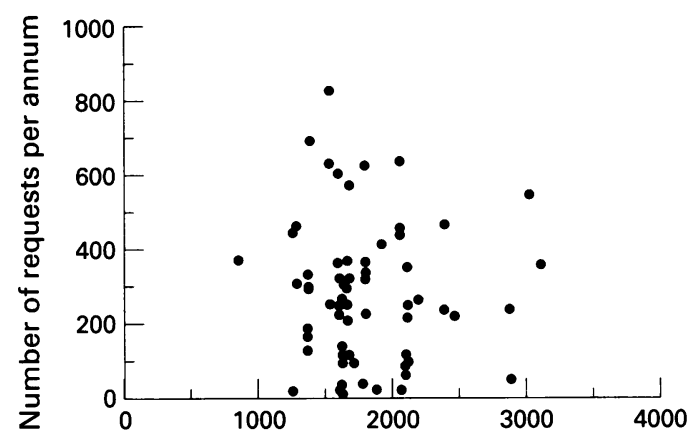

Number of patients per general practitioner

Figure 2 Data on the annual number of biochemistry requests plotted against the number of patients on the list for each general practitioner.

ing the total number of patients attending the practice by the number of general practitioners in the practice (in whole time equivalents). The data indicate a wide variation in usage between general practitioners, from no requests to over 800 per year, with no correlation between the number of requests and the size of general practitioner's list.

\section{TURNROUND TIME STUDY}

There were 10 respondents to the second survey (59\% response). The mean turnround time regarded as acceptable for a thyroid function test was 7.7 days, with a range from 2 to 10 days, although most general practitioners would like a $50 \%$ improvement in the figure. Of the 10 respondents, eight felt that problems lay with the frequency of the sample collection and results delivery service, four would prefer e-mail, one would prefer telephone, and one would prefer a direct modem link to the laboratory computer. A flow chart showing the progress of a typical thyroid function test sample request is shown in figure 3 , from observations of three analytical batches on separate days containing a number of samples from general practitioners. The preanalytical phase accounted for $41.7 \%$ of the total turnround

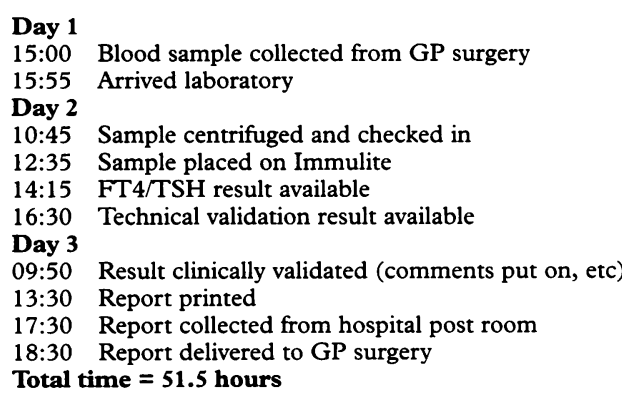

15:00 Blood sample collected from GP surgery

15:55 Arrived laboratory

Day 2

10:45 Sample centrifuged and checked in

12:35 Sample placed on Immulite

14:15 FT4/TSH result available

16:30 Technical validation result available

Day 3

09:50 Result clinically validated (comments put on, etc)

13:30 Report printed

17:30 Report collected from hospital post room

18:30 Report delivered to GP surgery

Total time $=\mathbf{5 1 . 5}$ hours

Figure 3 Flow chart showing the actual time taken for the production of thyroid function test results.

time, the analytical phase for $7.8 \%$, and the postanalytical phase $50.5 \%$.

\section{Discussion}

The appropriate use of pathology tests lies at the foundation of good clinical practice in both the hospital and primary care setting. ${ }^{11}$ The improvement in laboratory analysers and communication systems, together with the availability of near patient testing facilities, now offers the doctor-particularly the general practitioner-greater opportunities for a more responsive pathology service; the health reforms have stimulated the general practitioner to focus more attention on what is needed rather than what is available.

While the respondents were reasonably satisfied with the service provided, there were several interesting observations which would help in designing an improved service to general practitioners. There was dissatisfaction with collection times, and particular concerns were expressed about the delivery of results, which were clearly not getting back to the requester within an acceptable time frame-a feature of the Audit Commission's report on pathology services and other independent studies. $^{3-6}$ This result was particularly surprising in the light of the frequency with which the analyses were actually performed by the laboratory. The complaints about sample collection times are common and in the main reflect the fact that the transport is used for collection and delivery of a range of items, for example reports and minor consumables. ${ }^{3}$ Unfortunately what is often not recognised is that collection times can have a significant effect on the work pattern of practice staff: demands to complete venesections early in the day may be quite incompatible with smooth patient flow.

The tests investigated were chosen to reflect those that may lead to a hospital admission (urgent), a routine diagnostic test (non-urgent, for example urea and electrolytes), a common endocrine test (thyroid function), a screening test (cholesterol), and a monitoring test 
$\left(\mathrm{HbA}_{\mathrm{lc}}\right)$. Invariably the usual response time fell short of what general practitioners considered to be acceptable, and this observation led to the second part of the survey. The repertoire of investigations requested by the practitioners in this study is fairly typical in breadth and relative proportions, being a mixture of routine investigation, screening tests, and specific tests associated with the monitoring of chronic diseases, and showing the relatively high proportion of endocrine tests (particularly thyroid function) compared with electrolyte and liver function tests. What is more surprising was the highly variable rate of requesting among this cohort of general practitioners. Factors that influence the level of requesting may include: (1) the general health of the practice population; (2) the proportion of very young or elderly patients in the practice; (3) the expectations of the population within the practice; (4) the age and experience of the general practitioner; (5) the availability of special clinics in the practice-for example, diabetes, well person screening, hypertension, antenatal, asthma; (6) the presence of trainees in the practice; and (7) the quality of service provided by the laboratory. There have been several studies in which the variation in requesting pattern has been recognised; Henkley et $a l^{12}$ observed a wide variation in use of pathology but were unable to identify any relation between use and patient characteristics. Wilkin et $a l^{13}$ observed that the requesting pattern related more to the characteristics of the doctor than the patient. Mills and Reilly ${ }^{14}$ observed that part time general practitioners requested more tests on average than trainees, and full time practitioners had the lowest investigation rate. Batstone and Firstbrook, ${ }^{15}$ in a review of general practitioner requests sent to a laboratory, predicted trends in use and identified increasing demands for tests associated with the monitoring of chronic disease (for example diabetes) or for screening. They pointed out the need for consultation between general practitioners, laboratory directors, and purchasers to ensure a more appropriate use of services through agreement on requesting protocols. The question needs to be raised, therefore, whether in this study, where the majority of general practitioners work in a deprived inner city area and presumably see a similar mix of cases, the extremes of use that we found reflect underutilisation of services at one extreme and excessive use at the other.

The data gathered from the study of factors affecting the turnround time of results provided insight into the delays in producing results for general practitioners. The measurement of test turnround times has often been recommended as part of a laboratory's quality assurance programme,${ }^{16}{ }^{17}$ turnround time usually being defined as the time between specimen receipt in the laboratory and the reporting of the result. In the case of samples requested by general practitioners, our study and another ${ }^{18}$ have shown that the definition of turnround time needs to be broadened to cover the additional steps necessary before sample processing and following result reporting. In our study we found that the analysis time was disproportionately small and the major delays occurred in the transport of the specimen to the laboratory and in delivering the result back to the general practitioner. Smellie et $a l^{18}$ recently assessed turnround times for routine main analyser tests and found that the preanalytical phase accounted for $7-17 \%$, and the postanalytical phase $64-88 \%$ of total turnround time. Like them, we found that the system of sample collection resulted in deliveries to the laboratory which were too late for same day analysis. Considerable attention needs to be paid by laboratory staff to result reporting to the primary care sector. Although introduction of courier services may have advantages over postal delivery, ${ }^{18}$ modern communication systems such as e-mail or laboratory computer modem links will undoubtedly improve this further.

The turnround times for endocrine analyses which we found in this study fell within the expectations of the majority of general practitioners and although there are improvements which could be instituted to speed up the process, the medico-economic impact of faster turnround times remains to be established. ${ }^{19}$ However, it is also recognised that expectations will be influenced by experience, and improvement will often enhance expectation. Further studies are needed to establish whether faster turnround times influence patient management, outcome, or costs in the primary care sector.

Expectations on the turnround times for tests would suggest there is little likelihood of near patient testing bringing any additional benefit to the general practitioner unless it proves to be cheaper. However, this may be a slightly unfair conclusion as near patient testing was not offered as an option to the respondents in this survey. The study of near patient testing in general practice conducted by Rink et al showed for a limited range of tests that there were very few benefits to introducing testing facilities in the health centre. Indeed, there was a suggestion that the availability of testing on site might lead to more tests being requested and an increase in costs. Hobbs ${ }^{2}$ takes a contrasting view, predicting that near patient testing will bring benefits to primary care.

This study provides valuable data on the expectations of general practitioners in relation to the organisation of a clinical biochemistry service and some things that can be done to help to meet these expectations. The study indicates a wide variation of use among general practitioners, which may be related to the nature of the practice population. It poses questions about whether improving the response times will lead to increased use of the service and a need for changes in the organisation of the practice, and whether improved patient care can justify the increased investment.

We thank the general practitioners of the London Borough of Tower Hamlets who gave so willingly of their time to participate
in this study. 
1 Secretaries of State for Health, Wales, Northern Ireland and Scotland. Promoting better health. The government's programme for improving primary healthcare. London: HMSO, grami

2 Hobbs R. Near patient testing in primary care. BMf 1996; 312:263-4.

3 Audit Commission. Critical path. An analysis of the pathology services. London: HMSO, 1993.

4 Broom PS, Begley JP. Users' views of the East Dorset Clinical Biochemistry Service: a survey by questionnaire. Commun Lab Med 1992;4:73-6.

5 Paterson JR, Murphy MJ, Langan J, Dryburgh FJ, Shepherd J. "What do you think of the service?" An audit of local general practitioner requirements. Bull $R$ Coll Pathol 1992; 77:15-16.

6 Allen KR, Harris CM. Measure of satisfaction of general practitioners with the chemical pathology services in Leeds
Western Health District. Ann Clin Biochem 1992;29:331-6.

7 Kricka LJ. Trends in immunoassay technologies. F Clin Immunoassay 1993;16:267-71.

8 Hilton S. Near patient testing in general practice: a review. Br F Gen Pract 1990;40:32-6.

9 Rink E, Hilton S, Szczepura A, Fletcher J, Sibbald B, Davies $\mathrm{C}$, et al. Impact of introducing near patient testing for standard investigations in general practice. $B M \mathcal{F} 1993 ; 307$ : 775-8.

10 Lamont J. General practice pathology: a field trial of the Seralyzer. Aust Fam Physician 1988;17:547-8.

11 Grob P. General practitioners office. In: Marks V, KGMM
Alberti KGMM, eds. Biochemistry nearer the patient. Edinburgh: Churchill Livingstone, 1985:195-9.

12 Henkley RM, Charlton JR, Harris CM, Jarman B. Influence of patient characteristics on test ordering in general practice. BMF 1984;289:735-8.

13 Wilkin D, Metcalfe DHM, Hallam L, Cooke M, Hodgkin $P K$. Area variations in the process of care in urban general practice. $B M \mathcal{F}$ 1984;289:229-32.

14 Mills KA, Reilly PM. Laboratory and radiological investigations in general practice. BMF 1983;287:1033-6.

15 Batstone GF, Firstbrook AH. General practitioner users of pathology services. In: Griffin J, ed. Factors influencing clinical decisions in general practice. London: Office of Health cal decisions in general prac
Economics, 1990:41-8.

16 Hilborne LH, Oye RK, McArole JE, Repinski JA, Rodgerson DO. Use of specimen turnround time as a component of laboratory quality - a comparison of clinician expectations with laboratory performance. Am f Clin Pathol 1989; 92:613-18.

17 Howanitz PJ, Steindel SJ. Intralaboratory performance and laboratorians' expectations for stat turnround times. Arch Pathol Lab Med 1991;115:977-83.

18 Smellie WSA, Galloway PJ, Johnston KI. Laboratory turnround time: closing the loop. $\mathcal{F}$ Clin Pathol 1995;48: 372-5.

19 Valenstein PN. Turnround time-can satisfy clinicians' demand for faster service? Should we try? [Editorial] $A m \mathcal{F}$ Clin Pathol 1989;92:705-6. 